\section{Modelo de convivencia escolar. Un marco para políticas públicas, formación e investigación}

\section{A School Convivencia (peaceful coexistence) Model. A framework for public policy, education, and research}

\author{
Cecilia Fierro-Evans* \\ PATRICIA CARBAJAL-PADILLA**
}

\begin{abstract}
Este trabajo reporta el proceso de análisis documental realizado para responder la solicitud de la Secretaría de Educación de Guanajuato (México) de elaborar un modelo para atender, prevenir y erradicar la violencia escolar. El análisis de documentos de política pública, a la luz de la teoría de convivencia, permitió poner en diálogo dos enfoques distintos: uno sobre prevención de la violencia, que desemboca finalmente en el control de comportamientos antisociales, y otro de convivencia, orientado a la creación de ambientes enriquecedores y desarrollo de capacidades. Fue relevante identificar principios de fondo compartidos y nociones equivalentes desde ambas perspectivas. Asimismo, se observaron algunas divergencias, vacíos e inconsistencias que fueron atendidos. El modelo propuesto desplazó la violencia como eje de la política pública para situar la convivencia como núcleo principal de atención. Recuperando aportaciones de la sociología, la filosofía, la teoría de conflictos en escuelas, la pedagogía y la gestión escolar, el modelo de convivencia resultante propone tres niveles de intervención: contención, resolución de conflictos y transformación de prácticas pedagógicas y de gestión orientadas hacia la inclusión, la equidad y la participación. El modelo representa un dispositivo accesible para analizar políticas públicas, elaborar autodiagnósticos escolares y diseñar propuestas de formación en convivencia o llevar a cabo investigaciones.
\end{abstract}

Recibido: 22 de diciembre de 2020| Aceptado para su publicación: 24 de agosto de 2021| Publicado: 10 de septiembre de 2021

Cómo citar: Fierro-Evans, M. C. y Carbajal-Padilla, P. (2021). Modelo de convivencia escolar. Un marco para políticas públicas, formación e investigación. Sinéctica, Revista Electrónica de Educación, (57), e1242. https://doi.org/10.31391/S2007-7033(2021)0057-011 
This paper describes the process of documentary analysis to meet the request of the Ministry of Education of Guanajuato (Mexico) to develop a Model to Address, Prevent and Eradicate School Violence. The analysis of public policy documents, in the light of the theory of Convivencia (peaceful coexistence), allowed for a dialogue between two different approaches: One on violence prevention, which ultimately leads to the control of antisocial behaviors, and another on Convivencia, more oriented to the creation of nurturing environments and capacity building. It was relevant to identify shared basic principles and equivalent notions from both perspectives. We also observed some divergences, gaps and inconsistencies that were addressed. The proposed Model shifted the focus of public policy from school violence to convivencia as the focus of attention. Based on contributions from sociology, philosophy, conflict theory in schools, pedagogy and school organization, the resulting Convivencia Model proposes three levels of intervention: Containment, Conflict Resolution and Transformation of Pedagogical and Organizational Practices oriented towards inclusion, equity, and participation. The model represents an accessible resource for analyzing public policies, elaborating school self-diagnoses, designing convivencia training proposals or conducting research.
Keywords: convivencia (peaceful coexistence), school violence, public policies

\footnotetext{
* Doctora en Ciencias con especialidad en Investigación Educativa por el DIE-CINVESTAV-IPN. Académica numeraria de Universidad Iberoamericana León, adscrita al Departamento de Investigación y Posgrado. Líneas de investigación: prácticas docentes, formación de docentes en ejercicio, gestión, ética de la escuela y convivencia escolar. Correo electrónico: cecilia.fierro@iberoleon.mx/https:// orcid.org/0000-0002-9644-7637

**.Doctora en Estudios Curriculares y Desarrollo Docente con especialidad en Educación Comparada Internacional por el Ontario Institute for Studies in Education of the University of Toronto. Actualmente, se desempeña como consultora independiente y coordinadora del Seminario de Investigación en Convivencia Escolar de la Universidad Iberoamericana León. Líneas de investigación: educación para la paz y los derechos humanos, educación en valores, violencia y convivencia escolar. Correo electrónico: patricia.carbajalp2019@gmail.com/https:// https://orcid.org/0000-0002-3231-4456
} 


\section{INTRODUCCIÓN}

$\mathrm{E}$ l aumento de la violencia social y la manera en que se ha publicitado en los medios de comunicación han dado lugar a una creciente preocupación en el sector educativo, así como en las familias, a propósito de lo que acontece en las escuelas. De esta manera, el tema de "seguridad escolar" ha estado presente en la conversación de la vida diaria de las escuelas.

Las políticas públicas en materia de prevención de violencia y convivencia han tratado de dar respuesta a esta problemática. Sin embargo, los paradigmas con los que se han abordado han sido objeto de debate. De acuerdo con Magendzo, Toledo y Gutiérrez (2013), en temas de política pública referida a seguridad se ha dado un fenómeno de superposición de dos enfoques no solamente distintos, sino que responden a paradigmas antagónicos. El paradigma de seguridad pública pone el acento sobre el control y la sanción, mientras que el de seguridad humana refiere a la necesidad de crear las condiciones para establecer una convivencia escolar democrática, que respete los derechos humanos de los miembros de la comunidad educativa.

Diversos sectores, tanto académicos como de la sociedad civil, han manifestado distintas preocupaciones a propósito de las orientaciones predominantes de la política pública, como hemos discutido en otro espacio (Fierro, 2017), y que podemos resumir como sigue:

- Una deliberada confusión de términos para referirse a convivencia y violencia parece estar detrás de discursos que, luego de invocar la construcción de una cultura de paz, se decantan por una lógica punitiva, la cual es, en última instancia, la que orientará la operación cotidiana de las escuelas. En consecuencia, la lógica "dura" se impone sobre la "blanda"; es decir, las orientaciones formativas quedan relegadas en la actuación de docentes y directivos, toda vez que la aplicación u omisión de sanciones afecta su trayectoria laboral.

-Estas orientaciones de políticas han tenido como consecuencia no prevista el violar o limitar los derechos humanos al criminalizar las diferencias sociales, estigmatizar, segregar y discriminar a grupos de estudiantes, los cuales son las principales víctimas de la violencia.

- La forma en que opera el conjunto de protocolos y reglamentos derivados de políticas públicas enfocadas en la seguridad y prevención del crimen ha tenido el efecto de recargar aún más las tareas administrativas de docentes y directivos al tener que registrar todos los comportamientos antisociales en bitácoras, además de los procesos de seguimiento de cada caso, todo lo cual va en detrimento de su dedicación y tiempo para el quehacer educativo.

- De cara a la formación para la ciudadanía, los enfoques de convivencia basados en la seguridad escolar reducen de modo muy significativo las oportunidades para desarrollar capacidades democráticas en los estudiantes, ya que destacan la subordinación y el temor a la autoridad.

- El seguimiento de protocolos que judicializan los conflictos escolares establece per se una dinámica de desconfianza en la relación entre alumnos, 
docentes y padres de familia, lo cual atenta de manera inevitable y grave contra el vínculo pedagógico, que es la unidad básica de los procesos del aprender y convivir en las escuelas.

Este reconocimiento de consecuencias no deseadas destaca la importancia de explorar otro tipo de estrategias que recuperen el conocimiento acumulado en distintos contextos, de ahí que la investigación represente una interlocución válida que pueda aportar solidez en la formulación de nuevas propuestas de intervención.

Tomando como base un concepto de convivencia que responda más a la línea de seguridad humana, a la vez que considere las preocupaciones de seguridad escolar que están presentes en la perspectiva de prevención de violencia, entendemos la convivencia como los procesos y los resultados de los esfuerzos por construir una paz duradera en las escuelas, a través de prácticas pedagógicas y de gestión: inclusivas, equitativas y participativas, y que atiendan de manera dialógica los conflictos (Fierro y Carbajal, 2019). Este concepto comprende los elementos principales que recuperamos de la teoría de convivencia.

Nuestro artículo tiene el propósito de reportar el proceso de investigación documental realizado para atender la solicitud recibida de parte de la Coordinación Transversal para la Convivencia y la Cultura de la Paz, de la Secretaría de Educación de Guanajuato (SEG), para desarrollar la propuesta teórica Modelo de Convivencia para Atender, Prevenir y Erradicar la Violencia Escolar (Carbajal y Fierro, 2020), el cual fue aprobado por el Consejo Estatal para Prevenir, Atender y Erradicar la Violencia en el Estado de Guanajuato, el 10 de julio de 2020.

\section{POLÍTICAS PÚBLICAS DE CONVIVENCIA ESCOLAR E INVESTIGACIÓN EDUCATIVA EN MÉXICO: ¿POSICIONES ENCONTRADAS?}

Las políticas públicas de convivencia en México fueron formuladas en sus inicios desde un enfoque de seguridad pública. El Programa Escuela Segura nació en 2007 como parte de la Estrategia Nacional de Prevención del Delito y Combate a la Delincuencia, con el lema "Limpiemos México". Concebido como parte de una estrategia de seguridad y de combate al crimen organizado, este programa arrancó a partir de un conjunto de lineamientos, los cuales, de acuerdo con el Programa de Promoción de la Reforma Educativa de América Latina, pueden definirse como "de carácter restringido", esto es, orientados a prevenir la violencia y a establecer la seguridad a través de imponer el control, la vigilancia y la aplicación de sanciones (PREAL, 2003).

Así, con distintos matices y énfasis según los periodos de gobierno, la perspectiva de prevención de violencia ha permeado las políticas de convivencia escolar establecidas en México en los últimos años, cuestión que ha dado lugar a críticas desde la academia y otros sectores (Zurita, 2013; Landeros y Chávez, 2015; Chávez-Romo et al., 2016; Carbajal, 2016; Morales y López, 2019).

El punto central de tensión entre investigadores y políticas de convivencia refiere la manera de posicionarse, ya sea desde la seguridad pública, o bien, desde la seguridad humana (Magendzo, Toledo y Gutiérrez, 2013). En este sentido, el gran desafío para la formulación de políticas de convivencia radica en la necesidad de identificar núcleos de comprensión de la problemática en común entre investigadores y 
tomadores de decisión para lograr arribar a propuestas aceptables desde ambas perspectivas. La necesidad de generar respuestas más adecuadas frente a una problemática que desborda muchas veces las capacidades de actuación de las escuelas se presenta como el núcleo de interés compartido.

El argumento principal que sostenemos en este trabajo, derivado de la experiencia que se analiza, es que es posible llegar a acuerdos entre una perspectiva de prevención de la violencia y de convivencia, a partir de la identificación de coincidencias y del diálogo sobre las divergencias entre ambas perspectivas.

\section{CONSTRUYENDO PUENTES ENTRE POLÍTICAS PÚBLICAS E INVESTIGACIÓN EN CONVIVENCIA ESCOLAR}

Para la elaboración del modelo de convivencia, retomamos los siguientes documentos de política pública:

- Modelo Escolar de Prevención de la Violencia y la Delincuencia (2013-2014), elaborado por la Dirección General de Política Criminal y Prevención del Delito, adscrita a la Subsecretaría de Prevención de la Secretaría de Seguridad Pública del Estado de Guanajuato. Por instrucciones de la Secretaría de Educación de Guanajuato, este documento fue tomado como referencia para la elaboración del modelo de convivencia, ya que en él se establece "un marco teórico sobre la violencia en el ámbito escolar, que permita proponer líneas de acción para la prevención, atención y erradicación de la violencia en los planteles escolares" (p. 5).

-Ley para una Convivencia Libre de Violencia en el Entorno Escolar para el Estado de Guanajuato y sus Municipios (Gobierno del Estado de Guanajuato, 2013), cuyo objetivo es "establecer las bases que permitan el establecimiento de las políticas públicas para prevenir, atender y erradicar la violencia en el entorno escolar, así como distribuir las competencias entre el Estado y los Municipios" (artículo 1ํ), para lo cual “se implementará el modelo para la prevención, atención y erradicación de la violencia escolar elaborado por el Consejo Estatal para Prevenir, Atender y Erradicar la Violencia en el Estado de Guanajuato, en los términos que dispone esa ley" (artículo $2^{\circ}$, último párrafo).

- Reglamento Escolar para una Convivencia en Paz del Estado de Guanajuato (2014), que integra "la estrategia fundamental a implementarse por la Secretaría de Educación de Guanajuato, la cual se orienta esencialmente a la prevención de la violencia escolar a través de emisión de disposiciones de orden y disciplina establecidas" en dicho reglamento (p. 3).

- Programa Nacional de Convivencia Escolar. Protocolos para la detección, prevención y actuación en situaciones de abuso sexual infantil, acoso escolar y maltrato en las escuelas de educación inicial y básica para el estado de Guanajuato, de la Secretaría de Educación Pública (SEP, 2017). En este programa también se presentan los factores de riesgo y los factores de protección "que disponen y pueden prevenir que una alumna o alumno resulte receptor [de algún tipo de violencia], o bien trascienda a generador, en la escuela" (p. 19). 
En los siguientes apartados presentaremos los momentos principales en el proceso de elaboración del Modelo de Convivencia para Atender, Prevenir y Erradicar la Violencia Escolar.

\section{PRIMER MOMENTO: IDENTIFICAR ELEMENTOS COINCIDENTES ENTRE LOS DOCUMENTOS DE POLÍTICA PÚBLICA Y LA TEORÍA DE CONVIVENCIA}

Este momento consistió en un análisis en profundidad de los documentos de política pública con el objeto de encontrar puntos de coincidencia entre estos y la teoría de convivencia escolar. A continuación, presentamos el detalle del análisis realizado alrededor de las grandes narrativas compartidas entre la política pública y la investigación: principios básicos compartidos y factores de protección como proceso de formación.

\section{Principios básicos compartidos}

El gran paraguas que permitió establecer el diálogo entre los documentos de política pública y los aportes desde la investigación en convivencia fue la referencia a los derechos humanos como plataforma de formación y acción para la construcción de una cultura de paz en el ámbito escolar que permita atender, prevenir y erradicar la violencia. Esta perspectiva está claramente expresada en el Modelo Escolar de Prevención de la Violencia y la Delincuencia (2013-2014), en el Programa Nacional de Convivencia Escolar (SEP, 2017), en la Ley para una Convivencia Libre de Violencia en el Entorno Escolar para el Estado de Guanajuato y sus Municipios (2013), así como en el reglamento (2014) que se desprende de esa ley. Esto implica que los cuatro documentos hacen hincapié en ciertos principios comunes: el respeto a la dignidad humana, el respeto a los derechos humanos, la no discriminación, la solidaridad, la cohesión social, la libertad, la justicia y el reconocimiento de la diversidad, entre otros.

En relación con el Modelo Escolar de Prevención de la Violencia y la Delincuencia (2013-2014), destacamos el siguiente párrafo:

Las intervenciones deben estar orientadas a detonar un proceso de reconstrucción de la solidaridad, reeducar en derechos humanos, fomentar la no discriminación, la no violencia, la cultura de paz, la resolución pacífica de los conflictos, la negociación asistida, la cultura de la legalidad y el fortalecimiento de la cultura ciudadana (deberes y derechos), esto a partir de intereses u objetivos compartidos en la comunidad, para lograr la reconstrucción o rehabilitación de un tejido social más virtuoso y cohesionado (p. 58).

El Programa Nacional de Convivencia Escolar (SEP, 2017) señala lo siguiente:

El Estado de Guanajuato tiene la obligación de prevenir la violencia en el ámbito escolar y en cada uno de sus espacios de intervención, debe promover, respetar y garantizar los derechos humanos de niñas, niños y adolescentes que asisten a las escuelas de Educación Básica en nuestra entidad. [...] Es por ello que se requiere generar ambientes escolares de paz, a través de la práctica de la confianza y el respeto a los derechos humanos, así como la creación de comunidades escolares capaces de promover la cohesión social, limitando e inhibiendo las conductas violentas, donde la discriminación, en cualquiera de sus manifestaciones, sea erradicada (SEP, 2017, p. 5). 
En lo referente a la Ley para una Convivencia Libre de Violencia en el Entorno Escolar para el Estado de Guanajuato y sus Municipios (2013), tenemos lo siguiente:

Artículo 2을 apartado 1 . Son fines de la presente Ley: Establecer los principios y criterios que, desde la perspectiva de una cultura de paz y protección de los derechos humanos, orienten el diseño e instrumentación de políticas públicas para prevenir, atender y erradicar la violencia en el entorno escolar.

Por su parte, el Reglamento Escolar para una Convivencia en la Paz del Estado de Guanajuato (2014) se orienta a la prevención de la violencia escolar a través de "emisión de disposiciones de orden y disciplina" que promuevan:

... el respeto a los derechos fundamentales del ser humano, la adopción de vivencias valores, actitudes, comportamientos y modos de vida, que tienen por objeto reafirmar y perfeccionar la educación que se imparte en el seno familiar, a fin de que, los educandos, desarrollen y fortalezcan relaciones interpersonales y mutuas basadas en el respeto de la persona, su dignidad, sus ideas y creencias, así como en la libertad, justicia, lealtad y responsabilidad (p. 3).

Como observamos, hay una coincidencia fundamental en cuanto a los principios básicos para referir a las condiciones que permiten construir la convivencia. Ahora bien, el esfuerzo por comprender la lógica de la política pública desde la óptica de prevención de la violencia nos llevó también a identificar, en dos documentos fundamentales -el Modelo Escolar de Prevención de la Violencia y la Delincuencia (2013-2014) y el Programa Nacional de Convivencia Escolar (SEP, 2017)-, los llamados "factores de protección contra la violencia" como un elemento central de la prevención.

\section{Factores de protección como proceso de formación}

Un segundo aspecto de coincidencia entre los documentos de política pública revisados y la teoría de convivencia son los "factores de protección". Por estos se entiende aquellas situaciones y habilidades que mitigan la violencia en las escuelas (SEP, 2017, p. 23). En estos documentos encontramos una amplia gama de elementos, no necesariamente articulados entre sí, que, de manera general, aluden a dos aspectos formativos centrales: los factores de protección individuales y los factores de protección sociales.

Los primeros destacan el desarrollo de la conciencia y la valoración de sí mismo a través la formación en habilidades socioemocionales que promueven el autocontrol, la autogestión (lograr metas venciendo obstáculos) y la asertividad, así como en las habilidades necesarias para abordar los conflictos de una manera constructiva (SEP, 2017). Por su parte, los segundos aluden, entre otros aspectos, a la capacidad de desarrollar relaciones comunitarias en el aula y la escuela basadas en la colaboración y la igualdad entre compañeros, y a la importancia de generar oportunidades para el progreso académico de todos los estudiantes, que les ofrezca horizontes de vida prometedores (SEP, 2017).

En específico, el Modelo Escolar de Prevención de la Violencia y la Delincuencia (2013-2014) refiere a la importancia de promover "el desarrollo social y emocional de las personas", lo que les permitirá a los alumnos "desarrollar todo su potencial" (p. 67). En este sentido, destaca la necesidad de generar experiencias 
en los estudiantes que posibiliten tanto el crecimiento educativo como el progreso académico (p. 48). Asimismo, anima la participación de todos los integrantes de la comunidad escolar en la toma de decisiones en asuntos de interés colectivo, "abogando por la resolución de conflictos por vías no violentas, tales como el diálogo y la negociación" (p. 58).

Respecto a la dimensión social de los factores de protección, el Programa Nacional de Convivencia Escolar (SEP, 2017) señala la necesidad de desarrollar en los alumnos capacidades para mantener relaciones positivas en el entorno escolar:

Se necesita de la habilidad de trabajar bien con los demás, de acompañar, de crear un ambiente fuerte, colaborativo y efectivo, lo que permite a las niñas, niños y adolescentes establecer relaciones satisfactorias con otras personas en igualdad. En esta dimensión destacan dos indicadores; el de habilidades sociales y la resolución de conflictos (SEP, 2017, p. 24).

El énfasis en la importancia de los factores de protección como un elemento clave de formación tanto personal como colectiva es un asunto muy relevante de coincidencia entre la teoría de convivencia y los documentos de política pública, lo que aportó los cimientos para desarrollar un modelo teórico que vinculara las nociones que provienen de ambas fuentes.

\section{SEGUNDO MOMENTO: IDENTIFICAR NOCIONES EQUIVALENTES}

El segundo momento consistió en el esfuerzo por encontrar equivalencias entre la teoría de convivencia escolar, entendida como la construcción de una paz duradera, y los "factores de prevención contra la violencia." Si bien dichos factores tienen una especial relevancia en los dos documentos seminales de política pública mencionados, estos tienden a ser definidos de manera ambigua. Mientras que el Modelo Escolar de Prevención de la Violencia y la Delincuencia (20132014) hace hincapié en la importancia de controlar los factores de riesgo con el objeto de evitar conductas antisociales (p. 45), el Programa Nacional de Convivencia Escolar (SEP, 2017) se refiere al "ámbito de la prevención y detección" en los siguientes términos:

Los procedimientos aplicables en los centros educativos para evitar, detener y disminuir el daño de conductas violentas y su impacto es atendiendo entre otros, al interés superior de la niñez, a los principios de debida diligencia y pro persona, garantizando el derecho a tener una vida libre de toda forma de violencia y a la integridad personal, con el fin de lograr las mejores condiciones de bienestar y el libre desarrollo de la personalidad de las niñas, niños y adolescentes de la comunidad educativa, que permitan la convivencia armónica, pacífica e inclusiva (SEP, 2017, p. 14).

De manera más específica, en ambos documentos los factores de prevención se conceptualizan, en gran medida, en función de los factores de protección (abordados en el segmento anterior). Según el Modelo Escolar de Prevención de la Violencia y la Delincuencia (2013-2014), "los programas preventivos buscan favorecer y apoyar los factores de protección” (p. 52). De manera similar, el Programa Nacional de Convivencia Escolar menciona lo siguiente: "Una estrategia de prevención implica la promoción de factores protectores que posibilitan el desarrollo de la dimensión personal y social, los valores éticos y morales" (SEP, 2007, p. 23). 
En el análisis de ambos documentos encontramos que los factores de prevención siguen los lineamientos propuestos por la Organización Mundial de la Salud (OMS), los cuales se clasifican en tres niveles: prevención primaria, secundaria y terciaria.

La prevención primaria refiere la creación de condiciones para evitar que se produzca algún factor que genere violencia. Se enfoca en "la construcción de ambientes de inclusión, igualdad, democracia, respeto, promoción de la tolerancia, creación y operación de redes de apoyo, así como la erradicación de prejuicios, estereotipos y cualquier tipo de discriminación" (SEP, 2017, p. 5).

La prevención secundaria se orienta a la identificación de situaciones de riesgo. Se toma como referencia lo señalado en el artículo 22, fracción II, del Reglamento Escolar para una Convivencia en la Paz del Estado de Guanajuato, el cual estipula lo siguiente: "Compete al director, supervisor o jefe de sector y es aquella destinada a personas y grupos en donde se detecta el riesgo de convertirse en receptores o generadores de la violencia mediante la aplicación de medidas y acciones que tienden a evitar el surgimiento de posibles conflictos" (SEP, 2017, p. 6).

La prevención terciaria tiene que ver con la atención de situaciones en las cuales la violencia ya se hizo presente. Se hace mención del artículo 22, fracción III, del citado reglamento escolar: "Compete a las autoridades establecidas en la Ley y es aquella centrada en las personas que han sido receptoras o generadoras de la violencia, enfocándose en acciones para prevenir y erradicar la reincidencia, en el caso de las generaciones y la reproducción en el caso de los receptores" (SEP, 2017, p. 6).

En esta clasificación observamos cómo se transita de la prevención primaria basada en la construcción de ambientes de inclusión, igualdad y democracia (entre otros), la cual responde a una concepción de prevención más cercana a la idea de seguridad humana, hacia la prevención secundaria y terciaria, que se centran en identificar y atender las conductas violentas de los estudiantes. Estas últimas se acercan más al concepto de seguridad pública.

Para fines de la elaboración del modelo de convivencia, nos dimos a la tarea de reorganizar y equilibrar tanto los tres tipos de prevención antes mencionados como otros elementos incluidos en los factores protección previamente identificados en las coincidencias, y los integramos en tres niveles: atención, prevención y erradicación de la violencia escolar.

Así, en el primer nivel, al que llamamos "Atender la violencia escolar," incluimos la prevención secundaria y la prevención terciaria. En el segundo nivel, "Prevenir la violencia escolar", incorporamos los factores de protección que implicaban el desarrollo de habilidades socioafectivas y comunicativas, las cuales apoyan el fomento de capacidades para un manejo de conflictos por medios no violentos.

Finalmente, en el tercer nivel, "Erradicar la violencia escolar," integramos la prevención primaria y los factores de protección que aluden a la construcción de ambientes de inclusión, igualdad, democracia, respeto, promoción de la tolerancia, creación y operación de redes de apoyo, así como la erradicación de prejuicios, estereotipos y cualquier tipo de discriminación.

El aporte en esta etapa de la investigación fue proponer tres categorías que ordenaran la gran cantidad de principios estipulados en los documentos oficiales, de 
manera que quedara claramente establecida la diferencia entre un nivel amplio de atención que se orienta a construir las condiciones o ambientes a partir de prácticas pedagógicas y de gestión inclusivas, equitativas y participativas. Un segundo nivel que recupera los factores de protección entendidos como elementos formativos. Y un tercer nivel que considera la aplicación de reglamentos y protocolos orientados a atender aquellos comportamientos que hayan escalado a violencia.

El resultado de este ejercicio nos permitió definir la estructura o esqueleto del modelo de convivencia en tres niveles de intervención (ver tabla 1).

Tabla 1. Equivalencia entre modelo OMS, SEG y teoría de convivencia

\begin{tabular}{|c|c|c|}
\hline $\begin{array}{c}\text { Lineamientos propuestos } \\
\text { por la OMS }\end{array}$ & $\begin{array}{c}\text { Niveles de actuación solicitados } \\
\text { por SEG en el modelo }\end{array}$ & Teoría de convivencia \\
\hline Prevención primaria & Erradicación & $\begin{array}{c}\text { Transformación de prácticas } \\
\text { pedagógicas y de gestión }\end{array}$ \\
\hline Prevención secundaria & Prevención & Resolución de conflictos \\
\hline Prevención terciaria & Atención & Contención \\
\hline
\end{tabular}

Esta articulación la expresamos en la figura 1, que refleja los niveles de intervención del modelo.

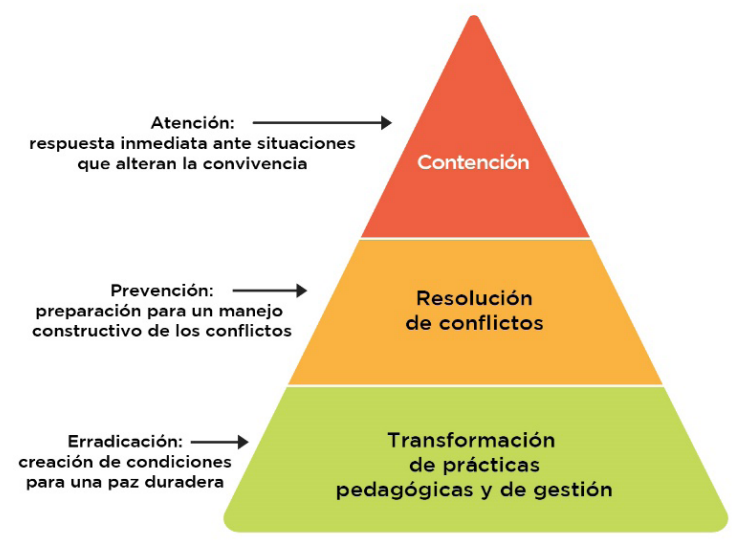

Figura 1. Niveles de intervención del modelo de convivencia

En resumen, el ejercicio de identificación de nociones equivalentes fue un paso fundamental en el proceso de diálogo entre la teoría de convivencia y los documentos de política pública. Como señalamos, incluyó la organización de un conjunto de elementos dispersos y de distinto alcance pedagógico en tres núcleos de intervención. Con esta medida, intentamos evitar el riesgo de atomizar las acciones propuestas en los documentos de política pública, lo que podría confundir a autoridades y docentes respecto de la importancia y alcance de unas y otras. 


\section{TERCER MOMENTO: IDENTIFICACIÓN DE VACÍOS, INCONSISTENCIAS O DISCREPANCIAS}

El análisis de los documentos de política pública fue tomando un giro más detallado conforme se definieron los tres niveles de intervención del modelo solicitado para la atención, prevención y erradicación de la violencia escolar. También fue posible identificar principios compartidos y equivalencias, y fuimos observando algunos vacíos existentes, inconsistencias y discrepancias, incluso, de cara a la teoría de convivencia escolar entendida como paz duradera.

\section{Vacíos}

El vacío más importante identificado en el nivel de contención (atención) es el relativo a la escasa consideración de sanciones que tengan claramente un enfoque formativo. En los casos de situaciones de violencia, la investigación considera necesaria la intervención de la autoridad para frenar las conductas agresivas de los alumnos con el objeto de evitar que causen daño a otras personas o a sí mismos. Sin embargo, dicha intervención debe privilegiar el aspecto formativo sobre el coercitivo.

En este sentido, observamos que el Modelo Escolar de Prevención de la Violencia y la Delincuencia (2013-2014) pone un especial acento en tres mecanismos de control: la elaboración de protocolos de atención ante situaciones de violencia que se puedan dar en la escuela; la elaboración de reglamentos que permitan la intervención rápida tanto en situaciones de disrupción (consideradas como formas leves de violencia) para así impedir conductas violentas de mayor gravedad; y el incremento de la vigilancia de los alumnos en espacios comunes con participación del personal docente a fin de evitar áreas en las que puedan darse situaciones de violencia.

Como veremos más adelante, parte de nuestra función fue tender hacia un balance entre los elementos que promueven el control de los comportamientos de los estudiantes y los elementos pedagógicos mencionados en los principios básicos compartidos, los cuales dan un claro sustento a la generación de propuestas formativas que complementen las medidas disciplinarias ya establecidas.

En este sentido, cabe señalar que las sanciones llamadas "formativas" en el reglamento escolar citado presentan limitaciones en su concepción y, además, tienden a desembocar tarde o temprano en la suspensión y la expulsión, sea debido a la reincidencia en la comisión de faltas menores, o bien, a faltas más graves. Veamos algunos ejemplos:

Artículo 84.... En caso de reincidencia en la falta cometida, el educando realizará alguna actividad educativa formativa adicional, asignada por el director y relacionada con la falta cometida:

IV. Servicio en favor de la comunidad educativa con autorización de los padres de familia: actividad formativa en mejora de las instalaciones en el servicio educativo, siempre y cuando, sea acorde a su edad y no ponga en riesgo su integridad física;

V. Separación temporal de la actividad escolar o clase: de uno a cinco días, sujeto al desempeño de una actividad con enfoque pedagógico que se le asigne y a la orientación y vigilancia del padre de familia o representante educativo; y 
VI. Separación de manera definitiva de la institución educativa: sin que esto constituya una privación del derecho a la educación, ni su exclusión del Sistema Educativo Estatal y Nacional. En este supuesto, la Secretaría, mediante las Delegaciones Regionales de Educación, ofertará a los padres o representantes educativos, las diversas alternativas escolares disponibles para que el educando continúe recibiendo el servicio público de educación (Reglamento Escolar para una Convivencia en la Paz en el Estado de Guanajuato, p. 40).

Llama la atención que, si bien este reglamento presenta la alternativa de "un servicio en favor de la comunidad educativa" como una actividad formativa, existe el riesgo de que ese servicio esté totalmente desvinculado de la falta cometida y, además, envía el mensaje de que acciones tan importantes como es un servicio a la comunidad sean catalogadas como castigo (Fierro et al., 2019).

En consecuencia, el vacío a ser atendido en este aspecto tiene que ver con ofrecer oportunidades a los estudiantes de reflexionar acerca de las consecuencias de sus acciones, así como de participar en la reparación del daño cometido e implicarse en acciones prosociales en favor de sus compañeros y la comunidad.

En el segundo nivel de resolución de conflictos (prevención) ubicamos los elementos formativos mencionados en los documentos de política pública como factores de protección, por ejemplo, el desarrollo de habilidades socioafectivas y comunicativas, así como de capacidades para un manejo no violento de los conflictos (Ley para una Convivencia Libre de Violencia en el Entorno Escolar para el Estado de Guanajuato y sus Municipios, 2013; Modelo Escolar de Prevención de la Violencia y la Delincuencia, 2013-2014; SEP, 2017).

En este nivel de prevención podemos identificar líneas claramente establecidas desde la política educativa. Tal es el caso de la nueva asignatura de Desarrollo socioemocional y la consideración de incorporar procesos de mediación escolar. Sin embargo, esta última intervención queda disminuida, ya que el Reglamento Escolar para una Convivencia en la Paz en el Estado de Guanajuato (2014) especifica algunas restricciones:

Art. 62. Sólo pueden ser objeto de mediación y conciliación los conflictos que no constituyan violencia escolar en los términos de la ley y su reglamento (p. 33).

Artículo 65. El mediador o conciliador escolar que colabore en la resolución de conflictos podrá ser un miembro del personal de apoyo, docente y directivo, padre de familia o cualquier integrante de la comunidad educativa designado, previa y expresamente, por el Organismo Escolar con la salvedad de que un educando no podrá fungir como conciliador escolar en un conflicto (pp. 34-35).

Artículo 77. Si un educando o miembro de la comunidad educativa es reincidente en una conducta generadora de conflicto, se aplicarán las medidas disciplinarias a que se refiere este Reglamento y demás disposiciones normativas, sin que éste tenga derecho la mediación y conciliación escolar (p. 37).

En las referencias citadas hay tres cuestiones importantes que resultan sumamente críticas desde el punto de vista de la convivencia. La primera es negar la posibilidad de mediación en situaciones definidas como violencia escolar. Si bien algunos investigadores tienen reservas en recomendar la mediación en casos de acoso en los que existe un desequilibrio de poder entre los estudiantes involucrados, la evidencia muestra que un proceso de mediación conducido de manera adecuada es una 
alternativa muy efectiva tanto para sensibilizar al generador de violencia acerca del daño causado a la víctima como para desarrollar las capacidades necesarias en el receptor de violencia para contrarrestar la agresión (Bickmore, 2016). Se requiere, por tanto, reconsiderar ese criterio.

El segundo problema es que los mediadores o conciliadores son elegidos por la autoridad y no por las partes en conflicto, lo que puede restar eficacia a su actuación como mediadores. La tercera cuestión es la no consideración de los estudiantes como posibles mediadores, ya que la investigación internacional ha mostrado con consistencia que la mediación entre pares es muy efectiva en la disminución de incidentes de violencia (Comisión Económica para América Latina y el Caribe, Fondo de las Naciones Unidas para la Infancia, Oficina Regional de Educación para América Latina y el Caribe-Organización de las Naciones Unidas para la Educación, la Ciencia y la Cultura, 2009; Villanueva, Usó y Adrián 2013.). Esta omisión es un tema que debe revisarse.

En este sentido, nuestra recomendación frente a los vacíos antes señalados consideró integrar dos grandes líneas más de intervención en este nivel, que son la mediación entre pares y los círculos de paz:

- La mediación entre pares es una estrategia que consiste en que los mismos estudiantes participan como mediadores entre sus compañeros. Los estudiantes-mediadores son capacitados en las habilidades necesarias para ejercer ese rol: promueven la escucha activa y la toma de perspectiva entre los compañeros involucrados en un conflicto y los apoyan a que ellos mismos propongan alternativas para resolverlo. Estos programas de mediación entre pares han tenido resultados muy positivos en diversos países. La investigación muestra que los niveles de indisciplina y violencia se reducen significativamente y que, además, mejoran los resultados académicos de los estudiantes involucrados (Jones, 2004).

- Los círculos de paz son una estrategia pedagógica orientada a facilitar el desarrollo de habilidades socioemocionales y comunicativas de todos los estudiantes de un grupo-clase para un manejo constructivo de los conflictos. Se basan en una forma de resolución colectiva de conflictos observada en pueblos originarios de distintos países del mundo, en donde se parte de la idea de que toda la comunidad se ve afectada cuando alguien entra en conflicto (Pranis, Stewart \& Wedge, 2003).

Una de las características principales de esta intervención formativa es su enfoque restaurativo, el cual consiste en reconocer que el estudiante que ha cometido una falta es miembro de una comunidad educativa, y que los miembros de esa misma comunidad le brindarán apoyo para que pueda comprender el daño que ha causado a los demás. Este enfoque considera que los comportamientos inadecuados no son únicamente faltas a un reglamento, sino que estos tienen consecuencias negativas en las relaciones interpersonales dentro del grupo, por lo que es importante hacer conscientes a los estudiantes de la necesidad de reparar el daño causado, así como de restaurar las relaciones entre los compañeros y reintegrar a la comunidad de aula o de la escuela a quienes han sufrido y ejercido violencia (Pulido, MartínSeoane y Lucas-Molina, 2013). El enfoque restaurativo ha mostrado tener un índice 
de reincidencia significativamente menor en comparación con el enfoque punitivo, que tiende a excluir a los infractores (Bickmore, 2016).

En síntesis, el proceso de análisis de documentos permitió observar asuntos relevantes que no estaban siendo considerados, y que las narrativas tanto de los principios básicos compartidos como de los factores de protección justificaban su inclusión. De ahí que en los tres niveles del modelo de convivencia tomamos en cuenta tanto aspectos ya incluidos en los documentos de política como otros que provienen de aportes de la investigación y que complementan lo establecido.

Discrepancias teóricas entre los documentos de política pública y la teoría de convivencia

El análisis documental permitió, además de identificar vacíos, observar algunas inconsistencias o contradicciones dentro de los mismos documentos revisados, cuya clarificación estimamos indispensable para fines de la elaboración del modelo de convivencia. Presentamos las más relevantes:

- Disrupciones como formas leves de violencia

La discrepancia teórica más importante encontrada, y la cual da lugar a otras más, es la manera de interpretar la relación entre violencia y conflicto. Una de las más frecuentes confusiones que encontramos en los documentos revisados es la idea de que el conflicto es en sí mismo una expresión de violencia o que, de manera inevitable, conduce a ella. Esta aproximación al conflicto ignora el hecho de que en cualquier espacio de interacción humana se presentan situaciones en las cuales entran en oposición necesidades, intereses, percepciones o valores, sean personales o grupales (Parra, 2016). En consecuencia, se precisan herramientas para abordarlos de un modo constructivo, es decir, como una oportunidad formativa para las personas implicadas.

Esta confusión se presenta en el Modelo Escolar de Prevención de la Violencia y la Delincuencia (2013-2014), que define disrupciones como formas no "especialmente graves" de violencia y no como manifestaciones de conflicto en el aula. Veamos la definición de disrupción que se ofrece:

La palabra disrupción se toma de las ciencias físicas, un disruptor es un dispositivo que se utiliza para provocar un estallido. En el plantel escolar, ciertas actitudes de los alumnos provocan un estallido de conflictos en las aulas, aunque estos comportamientos, no constituyen en sí mismos formas de violencia especialmente graves, son actitudes que cada día son más frecuentes en los planteles educativos. La disrupción por acumulación puede llegar a hacer imposible las actividades académicas, generan problemas importantes entre alumnos y profesores, ya que pueden ser causa de resentimiento y estrés, y base de algunos conflictos (Barri, 2011, citado en el Modelo Escolar de Prevención de Violencia y Delincuencia, 2013-2014, pp.15-16).

De acuerdo con la literatura sobre disciplina y convivencia (Furlán y Spitzer, 2013), las disrupciones refieren a las conductas de los estudiantes que interrumpen las tareas de clase, así como las normas de trabajo en el aula: guardar silencio, poner atención, permanecer sentados en su lugar y otras. Hay consenso en distintos autores en considerar la disrupción como resultado de la falta de interés o de motivación de los estudiantes por los contenidos curriculares o las pedagogías para abordarlos (Curwin y Mendler, 2003; Furlán y Spitzer, 2013; Ianni, 2003). Sin embargo, en el 
Modelo Escolar de Prevención de la Violencia y la Delincuencia (2013-2014) observamos una insistencia en equiparar las disrupciones con formas leves de violencia "llevadas a cabo por los alumnos, que van desde peleas, insultos, expresiones groseras, formas de desorden e indisciplina, exhibición de desmotivación, apatía, falta de cooperación, mala educación, insolencia, desobediencia, provocación, disposición hostil, amenazas, etc., mismas que afectan las normas de convivencia entre los miembros de la comunidad escolar y la tarea de los profesores” (p. 21).

Además de la confusión señalada en el sentido de equiparar la disrupción con la violencia, la cita anterior da cuenta de que asuntos de muy diversa índole son considerados como disrupciones. Algunos de ellos graves, como peleas y amenazas, otros relativos a la indisciplina, como la insolencia y la desobediencia; otros más que propiamente caben en la categoría de disrupción: el desorden y la falta de cooperación. La apatía y la falta de motivación están también aquí referidas, que son comportamientos que reflejan desinterés sin atentar contra las normas ni la tarea, por lo cual reflejan más bien la necesidad de un fortalecimiento pedagógico de parte de los docentes, lo que podría redundar en un aumento de la motivación y el sentido de tareas escolares.

Una grave consecuencia de equiparar las disrupciones con violencias se expresa en la indicación de elaborar protocolos para atenderlas: "Elaboración de protocolos de intervención con padres y profesores que les capaciten para actuar ante las conductas disruptivas" (Modelo Escolar de Prevención de la Violencia y la Delincuencia, 2013-2014, p. 69).

Si los docentes que ya reportan un fuerte desgaste con la aplicación de protocolos en casos graves de violencia, o bien, delitos, tuviesen que seguir protocolos ante las incontables disrupciones que pueden darse en las aulas, quedaría literalmente anulada su tarea pedagógica a favor del control y la sanción de los estudiantes. Por lo demás, resulta impensable involucrar a madres y padres de familia en la tarea de apoyar a los docentes frente a las conductas disruptivas -más aún definidas con la amplitud con la que se hace- que se presentan en clase.

- Vincula la idea de comportamientos violentos de estudiantes con delitos

Una consecuencia más que se desprende de la confusión entre conflicto y violencia consiste en vincular, de manera general, los comportamientos antisociales de los estudiantes con acciones que pueden desembocar en hechos delictivos. En este rubro, como en los anteriores, es constante la tendencia de nombrar y clasificar los comportamientos de los estudiantes en categorías que corresponden a acciones de mayor gravedad y mezclan, además, asuntos de diversa índole. Por ejemplo, en el rubro de "Lesiones" se incluyen comportamientos que van "desde golpes y empujones, hasta la utilización de armas punzocortantes y de fuego" (Modelo Escolar de Prevención de la Violencia y la Delincuencia, 2013-2014, p. 23). Esta cita presenta en la misma categoría faltas leves (como son los empujones), faltas graves (como golpes) y también delitos (por ejemplo, la utilización de armas punzocortantes y de fuego). Por ello, se hace necesario distinguir tres niveles de faltas: leves, graves y delitos. 
La confusión anterior tiene graves consecuencias al momento de formular reglamentos escolares y se expresará en la falta de correspondencia y proporcionalidad al establecer la relación entre faltas y sanciones, como se señaló en un estudio sobre reglamentos escolares de nivel básico (Landeros y Chávez, 2015), así como en el análisis de reglamentos escolares de media superior (Fierro et al., 2019). Así, "un empujón", que incluso podría ser por accidente, puede ser catalogado y sancionado, de acuerdo con estos lineamientos, como una falta grave o aun como un delito, debido a la incorrecta definición y clasificación de las faltas: "La importancia de revisar la correspondencia y la proporcionalidad entre faltas y sanciones se debe a que dichos aspectos implican establecer un trato justo a los estudiantes, que les permite asumir que sus acciones tienen consecuencias, pero que éstas se relacionan estrechamente con el tipo y la gravedad de la falta cometida" (Fierro et al., 2019, p. 59).

En síntesis, la discrepancia teórica fundamental que identificamos es una visión sobre los estudiantes y los conflictos que se presentan en el espacio escolar, como antesala cuasinecesaria de violencia escolar. Cualquier confrontación hacia la autoridad se aprecia como amenaza y, por tanto, como violencia (Kaplan, 2020). Esta visión se contrapone con una perspectiva que reconoce el conflicto como inherente a las relaciones humanas intergeneracionales y como oportunidad para desarrollar capacidades para la vida compartida.

Hasta aquí el proceso seguido en el análisis de los documentos de política, el cual consistió, principalmente, en recuperar y potenciar las coincidencias derivadas de las narrativas compartidas, así como las nociones equivalentes. Fue también relevante aprovechar el reconocimiento de omisiones en los documentos revisados, además de elaborar la argumentación teórica alrededor de las discrepancias identificadas en los propios documentos revisados, con vistas a llegar a acuerdos que permitieran subsanarlas.

De todo ello surge la propuesta de un modelo de convivencia que a continuación presentamos.

\section{Cuarto momento: elaboración del Modelo de Convivencia para ATENDER, Prevenir y ERradicar la Violencia Escolar}

Una de las consecuencias más importantes del proceso de análisis de documentos de política pública fue la posibilidad de modificar el centro de atención de un modelo para la atención, prevención y erradicación de la violencia escolar a favor de un modelo de convivencia. Esta formulación representa el más importante desplazamiento teórico resultado de este proceso, lo cual supone sostener que construir la convivencia es en sí misma la estrategia más robusta para atender, prevenir y erradicar la violencia escolar.

El resultado de este proceso de análisis es un modelo teórico, estructurado en tres niveles de análisis e intervención para la construcción de una cultura de paz en las escuelas:

-El primer nivel, llamado de contención, conocido en los documentos de política pública como atender la violencia escolar, refiere a la respuesta inmediata de parte de la autoridad ante las situaciones que alteran la convivencia. 
-El segundo se denomina resolución de los conflictos y corresponde al de prevenir la violencia en las escuelas. Se orienta a promover el desarrollo de capacidades y habilidades para enfrentar, de manera constructiva, los conflictos inherentes a la vida escolar.

-El tercero, corresponde al de transformar las prácticas pedagógicas y de gestión, el cual sienta las bases para la construcción de una paz duradera en las escuelas, de ahí que equivale a erradicar la violencia en el espacio escolar mediante la aplicación de prácticas de inclusión, equidad y participación.

Los espacios o ámbitos de intervención que prevé el modelo son también tres: pedagógico-curricular, que corresponde al trabajo del aula, por lo cual refiere las prácticas docentes y el trabajo con el grupo de clase; organizativo-administrativo, que tiene que ver con las prácticas directivas y docentes involucradas en los procesos de gestión institucional; y sociocomunitario, que considera la participación de padres y madres de familia, así como de otros miembros e instancias comunitarias que apoyan la labor de la escuela en favor del aprendizaje y la formación integral de los estudiantes.

\section{Concepto de convivencia y su base teórica}

El modelo se basa en un concepto de convivencia entendida como los procesos y resultados de los esfuerzos por construir una paz duradera entre los miembros de la comunidad escolar, a partir de prácticas pedagógicas y de gestión: inclusivas, equitativas y participativas que aborden de manera constructiva el conflicto (Fierro y Carbajal, 2019). Este concepto retoma los aportes de diversas líneas teóricas:

- Desde la sociología, el “triángulo de las violencias”, de Johan Galtung (1988), plantea que la violencia directa es la que se hace visible a través de los comportamientos de personas o grupos que causan daño o que cometen actos de destrucción contra otras personas o bienes; sin embargo, sostiene que detrás de la violencia visible y directa frecuentemente hay una violencia estructural, cuya característica fundamental es la desigualdad, la cual se reproduce generación tras generación. La violencia cultural, por su parte, es el conjunto de creencias, valores y actitudes que legitiman la violencia directa y la estructural. En otras palabras, la violencia cultural hace que el racismo, el sexismo y la homofobia, entre otros, así como el uso de la violencia hacia determinados grupos de personas, se vean como aceptables o racionales (Galtung, 1990, 2013).

Galtung (1969), reflexionando sobre este fenómeno, propuso también los conceptos de paz negativa o efímera y paz positiva o duradera para ayudar a comprender distintas formas de hacer frente a la violencia directa, a la violencia estructural y a la violencia cultural. El concepto de paz negativa alude a los esfuerzos por promover la ausencia de violencia directa, o alguna de sus manifestaciones -se trate de agresiones físicas o verbales o de las distintas formas de abuso físico o psicológico-, pero sin atender las causas que la generan. En cambio, la paz positiva implica no solo contener la violencia directa, sino también eliminar la violencia estructural, así como cuestionar y erradicar la violencia cultural. Sin embargo, construir la paz duradera no significa que desaparecen los conflictos, que son inherentes a la vida escolar y social en general, sino que se abordan de una manera constructiva. 
- Desde la teoría de conflictos, proponemos un clara distinción entre conflicto y violencia. Por conflicto entendemos todas aquellas situaciones en las que se presenta oposición en las percepciones, intereses, necesidades o valores entre las personas, grupos o Estados en relación con un objeto o idea en disputa (Parra, 2016). Cuando los conflictos se abordan adecuadamente, es posible llegar a una resolución no violenta de ellos. Sin embargo, cuando se enfrentan de una manera inadecuada, tarde o temprano darán lugar a formas de violencia directa. El manejo constructivo de los conflictos, que atienda las causas profundas que los originan, puede llevar a la transformación de las relaciones sociales, así como a la convivencia entendida como la construcción de una paz duradera.

Un aporte importante desde esta perspectiva proviene de Kathy Bickmore (2011), investigadora que ha enfocado su atención al análisis de los conflictos escolares, lo que le permitió identificar distintos modos de abordarlos, cada uno de las cuales ofrece mayores o menores oportunidades para construir una paz duradera en las escuelas. Los niveles que propone son peace keeping, peace making y peace buiding, los cuales renombramos, para fines del modelo de convivencia, como contención, resolución de conflictos y transformación de prácticas pedagógicas y de gestión, respectivamente.

- Desde la filosofía, Nancy Fraser (2003) aporta a la perspectiva sociológica de Galtung, al proponer tres dimensiones para el análisis y la construcción de la justicia social en sociedades contemporáneas:

-Reconocimiento: constituye la dimensión cultural de la justicia social.

-Redistribución: refiere la dimensión económica de la justicia social.

-Representación: esta dimensión política de la justicia social.

Esta perspectiva tridimensional de la justicia social permite examinar de una manera profunda distintos aspectos de la violencia estructural y la violencia cultural, con la finalidad de encontrar alternativas que lleven a la construcción de una paz duradera, es decir, a la construcción de una convivencia justa como forma de vida.

- Desde la pedagogía, Carbajal $(2018,2016)$ retoma tanto los aportes de Galtung (1969) y los conceptos de paz efímera y paz duradera como los niveles de intervención que propone Bickmore ( 2011) en el manejo de los conflictos escolares. Asimismo, de Fraser (2003) recupera el modelo tridimensional de la justicia social y lo adapta para orientar las prácticas pedagógicas en el aula con vistas a construir la convivencia, entendida como paz duradera. Las dimensiones de Fraser son replanteadas por Carbajal como inclusión (reconocimiento), equidad (redistribución) y participación (representación). 
Tabla 2. Adaptación a la educación del modelo tridimensional de justicia social para la construcción de la convivencia, entendida como paz duradera

\begin{tabular}{|c|c|c|}
\hline $\begin{array}{l}\text { Dimensión de la } \\
\text { justicia social }\end{array}$ & $\begin{array}{l}\text { Aspecto de la justicia } \\
\text { que privilegia }\end{array}$ & Adaptación a la educación \\
\hline Cultural & $\begin{array}{l}\text { Reconocimiento: implica la valoración de las } \\
\text { diversas identidades y necesidades básicas de } \\
\text { las personas, así como la toma de conciencia } \\
\text { de que ciertos individuos o grupos son o han } \\
\text { sido consistentemente considerados como } \\
\text { inferiores, excluidos o simplemente invisibles }\end{array}$ & $\begin{array}{l}\text { Inclusión: se orienta a reconocer las di- } \\
\text { versas necesidades e identidades de los } \\
\text { estudiantes con el propósito de crear } \\
\text { comunidad en el grupo de clase }\end{array}$ \\
\hline Económica & $\begin{array}{l}\text { Redistribución: alude al acceso y distribución } \\
\text { equitativa de recursos y de bienes, y genera } \\
\text { estrategias de atención que permitan reducir } \\
\text { las brechas en el estatus socioeconómico de los } \\
\text { sujetos derivadas de la violencia } \\
\text { estructural }\end{array}$ & $\begin{array}{l}\text { Equidad: se orienta a crear oportu- } \\
\text { nidades de acceso al conocimiento } \\
\text { como un recurso indispensable en las } \\
\text { sociedades contemporáneas a partir de } \\
\text { la construcción del andamiaje necesa- } \\
\text { rio para asegurar que cada uno de los } \\
\text { estudiantes -especialmente quienes } \\
\text { enfrentan mayor rezago académico- } \\
\text { puedan aprender }\end{array}$ \\
\hline Política & $\begin{array}{l}\text { Representación: refiere a las oportunidades } \\
\text { que los individuos y grupos tienen para expre- } \\
\text { sar sus propias voces y perspectivas para ser } \\
\text { escuchados y participar en términos equitati- } \\
\text { vos en procesos de toma de decisiones sobre } \\
\text { asuntos que les conciernen }\end{array}$ & $\begin{array}{l}\text { Participación: se orienta a fortalecer } \\
\text { el protagonismo de los estudiantes } \\
\text { para expresar su propia voz en asuntos } \\
\text { que les conciernen a nivel individual y } \\
\text { colectivo, así como en los procesos de } \\
\text { resolución de conflictos }\end{array}$ \\
\hline
\end{tabular}

Fuente: Adaptación de Carbajal, 2018, 2016.

- Finalmente, desde la perspectiva de la gestión, asumimos que las prácticas docentes y escolares están enraizadas tanto institucional como socioculturalmente; es decir, están condicionadas por el espacio social específico que es la escuela y por un momento histórico particular; por valores, significados, tradiciones y costumbres de la propia escuela, así como por el entorno local. Los estilos y prácticas pedagógicas y de gestión de directivos y docentes, de manera preponderante, son elementos que configuran procesos que pueden apuntar, o no, al aprender a vivir juntos.

En consecuencia, es posible plantear que la gestión escolar y sus ámbitos, es decir, los distintos espacios de la vida compartida en la escuela, referidos a lo pedagógicocurricular, lo organizativo-administrativo y lo sociocomunitario, son la unidad de análisis adecuada para reflexionar la contribución que cada escuela está haciendo en su día a día para construir la convivencia, y centrar la atención, la observación y la evaluación en las prácticas pedagógicas y organizativas (Hirmas y Carranza, 2009; Fierro y Tapia, 2013; Fierro et al., 2013). Esto significa que es posible plantearlas también como objetos de transformación, esto es, como prácticas que pueden ser intervenidas en función de que contribuyen a construir la convivencia, el aprender a vivir juntos.

El ámbito pedagógico-curricular remite a las prácticas docentes tanto en su manejo de aspectos didácticos como organizativos y relacionales, relativos a la planeación, desarrollo curricular y prácticas de enseñanza orientadas a propiciar la construcción de aprendizajes -tanto de orden disciplinar como formativos para la vida- por parte de los estudiantes. El ámbito organizativo-administrativo refiere a las prácticas directivas y docentes involucradas en los procesos de gestión institucional para la construcción de un proyecto educativo escolar. El ámbito sociocomunitario, a las prácticas y procesos de la gestión directiva y de los docentes, orientados a establecer vínculos con los padres de familia, con la 
comunidad externa en que se sitúa la escuela y con otras dependencias e instancias de apoyo al trabajo de la escuela (Fierro y Tapia, 2013).

Recapitulando lo expuesto hasta aquí, integramos los elementos anteriores en un modelo teórico que permita, de manera efectiva, orientar las acciones tanto en el nivel de política pública como de formación y actuación en las escuelas con base en criterios que reduzcan no solo los niveles de violencia, sino construir la convivencia, entendida como una paz duradera entre los miembros de la comunidad escolar (ver figura 2).

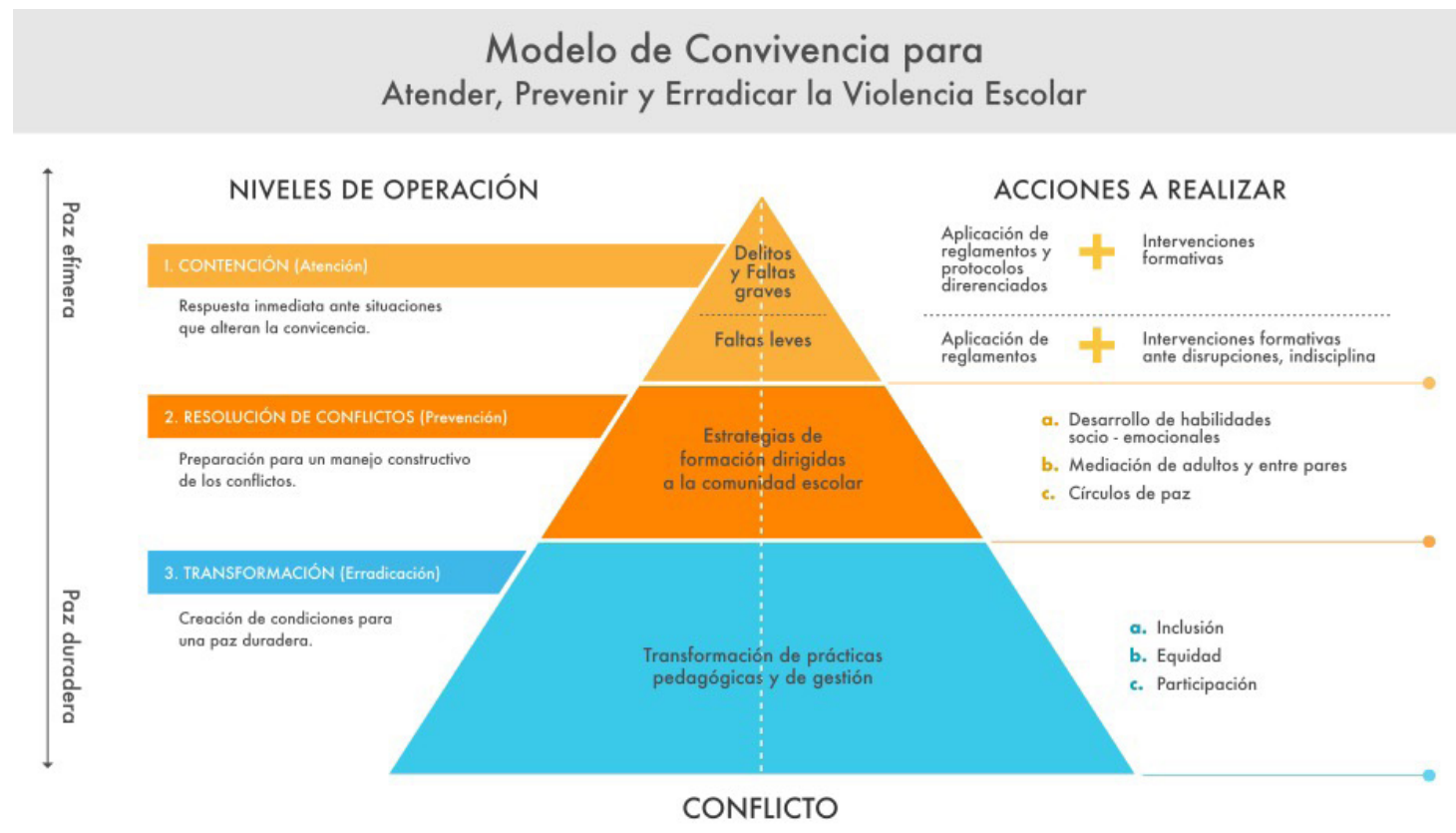

Figura 2. Modelo de Convivencia para Atender, Prevenir y Erradicar la Violencia Escolar. Fuente: Carbajal y Fierro (2020).

Este modelo teórico no se plantea únicamente como una herramienta para fines de política pública, sino también como un recurso que puede ser utilizado en las escuelas para analizar sus propios procesos en el sentido de diagnosticar y hacer propuestas; así también, puede orientar procesos de formación de agentes educativos y apoyar el desarrollo de proyectos de intervención o investigación.

\section{DISCUSIÓN Y REFLEXIONES FINALES}

El proceso de análisis reportado hasta aquí partió de una premisa: la diferencia esencial entre un modelo de prevención de violencia y uno de convivencia radica en que el enfoque de prevención de violencia, a pesar de que considera los principios y factores de protección que deben orientar las relaciones humanas en la escuela, en última instancia los relega a favor de la formulación de reglamentos y protocolos orientados al control de comportamientos antisociales.

En contraste, un modelo de convivencia se ocupa de ofrecer un marco amplio para el desarrollo de propuestas que permitan operacionalizar los principios básicos y los factores de protección mencionados en los documentos de política pública, a través 
de prácticas pedagógicas y de gestión que van permeando la vida entera de la escuela. Sostenemos que se va más allá del control de los comportamientos antisociales al promover la construcción de ambientes positivos de relación humana, en cuyo seno se desarrollan capacidades basadas en la aceptación de la diferencia del otro como un ejercicio de inclusión y pluralidad; en el abordaje de los conflictos de una manera constructiva, partiendo de la idea de que, además de inevitables en las relaciones humanas, no necesariamente desembocan en violencia. Asimismo, en asumir la solidaridad y el ejercicio de la colaboración como un principio básico de relación no únicamente a nivel interpersonal, sino también en las tareas académicas, con el objeto de que cada uno de los alumnos tenga la oportunidad de tener éxito en sus estudios. Todas estas habilidades serán puestas en juego no solo en el espacio escolar, sino en los distintos ámbitos de la vida social de los miembros de la comunidad escolar.

En síntesis, el Modelo de Convivencia para Atender, Prevenir y Erradicar la Violencia Escolar pretende ofrecer un dispositivo tanto de orden teórico como metodológico, dirigido a distintos actores del sistema educativo. De cara a la política pública, ofrece una herramienta para visualizar, de manera rápida, dónde están siendo colocados los programas y recursos orientados a mejorar la convivencia en las escuelas. Permite hacer un diagnóstico efectivo para contrastar necesidades frente a acciones en curso.

Como herramienta formativa dirigida a directivos y docentes, el modelo de convivencia ofrece la posibilidad de ampliar la mirada al reconocer que las acciones pedagógicas que están llevando a cabo para apoyar los aprendizajes de sus estudiantes, o animar su participación en las actividades de aula y la escuela, contribuyen a construir convivencia.

Finalmente, el modelo puede orientar también tareas de investigación de procesos y prácticas de convivencia, así como el seguimiento de proyectos de intervención y mejora en las escuelas.

La experiencia de investigación documental reportada en este artículo muestra, en última instancia, un esfuerzo orientado a poner en diálogo dos perspectivas distintas: una sobre la prevención de la violencia, enfocada en la seguridad pública y la otra sobre convivencia escolar, basada en la noción de seguridad humana, las cuales, a simple vista, podrían parecer como antagónicas. Sin embargo, al analizarlas en profundidad, fue posible identificar coincidencias de fondo que permitieron construir esta propuesta.

\section{REFERENCIAS BIBLIOGRÁFICAS}

Bickmore, K. (2016). Políticas y programas para escuelas más seguras: ¿las medidas "contra el bullying" obstruyen a la educación para la construcción de paz? Revista Iberoamericana de Evaluación Educativa, vol. 6, núm. 2, pp. 3771. https://revistas.uam.es/riee/article/view/3404

Bickmore, K. (2011). Keeping, making, and building peace in school. Social Education, vol. 75, núm. 1, pp. 40-44. https://www.socialstudies.org/system/files/publications/articles/se_75011140.pdf

Carbajal, P. (2018). Building democratic convivencia (peaceful coexistence) in classrooms. Case studies of teaching in Mexican public schools surrounded by violence. Tesis doctoral. Ontario Institute for Studies in Education, Universidad de Toronto. http://hdl.handle.net/1807/89835 
Carbajal, P. (2016). Educación para una convivencia democrática en las aulas. Tres dimensiones pedagógicas para su análisis. En N. Tello y A. Furlán (eds.). Violencia escolar: aportes para la comprensión de su complejidad (pp. 52-81). México: UNAM/SUIVE.

Carbajal, P. y Fierro, C. (2020). Modelo de Convivencia para Atender, Prevenir y Erradicar la Violencia Escolar. México: Universidad Iberoamericana-León/ Secretaría de Educación de Guanajuato.

Chávez-Romo, C., Gómez-Nashiky, A., Ochoa-Cervantes, A. y Zurita-Rivera, U. (2016). La Política Nacional de Convivencia Escolar de México y su impacto en la vida en las escuelas de educación básica. Posgrado y Sociedad. Revista Electrónica del Sistema de Estudios de Posgrado, vol. 14, núm. 1, pp. 2-13. https://doi.org/10.22458/rpys.v14i1.1483

Comisión Económica para América Latina y el Caribe, Fondo de las Naciones Unidas para la Infancia, Oficina Regional de Educación para América Latina y el Caribe-Organización de las Naciones Unidas para la Educación, la Ciencia y la Cultura (2009). Primer encuentro para la réplica en innovación social. La mediación, el secreto para prevenir la violencia escolar (pp. 21-25). Chile: CEPAL/UNICEF/OREALC-Unesco/Ministerio de Educación de Chile.

Curwin, R. y Mendler, A. (2003) Disciplina con dignidad. Guadalajara: ITESO.

Fierro, C. (2017). Escuelas y docentes en contextos de violencia y exclusión. Contribución a la construcción de tejido social. XIV Congreso Nacional de Investigación Educativa. Diálogos Magistrales (pp.127-147). San Luis Potosí: Consejo Mexicano de Investigación Educativa.

Fierro, C., Carbajal, P., Fortoul, B. y Pedroza, P. (2019). Análisis de reglamentos escolares en educación media superior. México: Instituto Nacional para la Evaluación de la Educación. https://www.inee.edu.mx/wp-content/ uploads/2019/04/P1D253.pdf

Fierro, C., Tapia, G., Fortoul, B., Martínez-Parente, R., Macouzet, M. y Jiménez, M.C. (2013). Conversando sobre la convivencia en la escuela: una guía para el auto-diagnóstico de la convivencia escolar desde las perspectivas. Revista Iberoamericana de Evaluación Educativa, vol. 6, núm. 2, pp. 103-124. https:// revistas.uam.es/riee/article/view/3407

Fierro, C. y Tapia, G. (2013). Hacia un concepto de convivencia escolar. En A. Furlán y T. Spitzer (coords.). Convivencia, disciplina y violencia en las escuelas 2002-2011 (pp. 74-87). México: COMIE/ANUIES.

Fierro-Evans, C. y Carbajal-Padilla, P. (2019). Convivencia escolar: una revisión del concepto. Psicoperspectivas, vol. 18, núm. 1, pp. 1-14. https://www.psicoperspectivas.cl/index.php/psicoperspectivas/article/view/1486/982

Fraser, N. (2003). Social justice in the age of identity politics: Redistribution, Recognition and Participation. En N. Fraser y A. Honneth. Redistribution or recognition? A political philosophical exchange (pp. 7-109). London, New York: Verso.

Furlán, A. y Spitzer, T. (coords.) (2013). Convivencia, disciplina y violencia en las escuelas 2002-2011. México: COMIE/ANUIES. Estados del Conocimiento

Galtung, J. (2013). Conflict transformation by peaceful means (the transcend method). En J. Galtung y D. Fischer. Johan Galtung, pioneer of peace research (pp. 59-70). Heidelberg, Nueva York, Dordrecht, Londres: Springer.

Galtung, J. (1990). Cultural violence. Journal of Peace Research, vol. 27, núm. 3, pp. 291-305. https://doi.org/10.1177/0022343390027003005

Galtung, J. (1988). Sobre la paz. Barcelona: Ed. Fontamara. 
Galtung, J. (1969).Violence, peace, and peace research.Journal of Peace Research, vol. 6, núm. 3, pp. 167-191. https://doi.org/10.1177/002234336900600301

Gobierno del Estado de Guanajuato (2014). Reglamento escolar para una convivencia en la paz en el estado de Guanajuato http://www.udec.edu.mx/Circulares-Documentos/ReglamentoEscolarConvivenciaEstado.pdf

Gobierno del Estado de Guanajuato (2013). Ley para una Convivencia Libre de Violencia en el Entorno Escolar para el Estado de Guanajuato y sus Municipios. egob.guanajuato.gob.mx:8088/files/FRAC_1/LEY_LOCAL/LEY_PARA_ UNA_CONVIVENCIA_LIBRE_DE_VIOLENCIA_EN_EL_ENTORNO_ESCOLAR_ PARA_EL_ESTADO_DE_GUANAJUATO_Y_SUS_MUNICIPIOS_JUL_2013.pdf

Gobierno del Estado de Guanajuato (2013-2014). Modelo Escolar de Prevención de la Violencia y la Delincuencia. Subsecretaría de Prevención, Dirección General de Política Criminal y Prevención del Delito, Secretaría de Seguridad Pública del Estado de Guanajuato. http://www.seg.guanajuato.gob.mx/ AConvivir/Paginas/dctos/Protocolo/ModeloEscolarPrevencionViolenciaDelincuencia.pdf

Hirmas, C. y Carranza, G. (2009). Matriz de indicadores sobre convivencia democrática y cultura de paz en la escuela. En Educación para la paz, la convivencia democrática y los derechos humanos. Santiago, Chile: OREALC/Unesco.

Ianni, N. (2003) La convivencia escolar: una tarea necesaria, posible y compleja. Monografías virtuales. Ciudadanía, democracia y valores en sociedades plurales. Organización de Estados Iberoamericanos para la Educación, la Ciencia y la Cultura, número 2, agosto-septiembre. http://www.oei.es/valores2/monografias/monografia02/reflexion02.htm

Jones, T. (2004). Conflict resolution education: The field, the findings, and the future. Conflict Resolution Quarterly, vol. 22, núm.1-2, pp. 233-267. http:// dx.doi.org/10.1002/crq.100

Kaplan, C. (2020). Violencias y emotividades en contextos escolares. Una relación de imbricación. En A. Furlán y N. E. Ochoa (coords.). Investigando sobre la convivencia y la violencia en las escuelas (pp. 211-223). Rosario: Homo Sapiens.

Landeros L. y Chávez, C. (2015). Convivencia y disciplina en la escuela. Análisis de reglamentos escolares de México. México: Instituto Nacional de la Evaluación de la Educación. http://publicaciones.inee.edu.mx/buscadorPub/ P1/C/232/P1C232.pdf

Magendzo, A., Toledo, M. y Gutiérrez, V. (2013). Descripción y análisis de la Ley sobre Violencia Escolar (№20.536): dos paradigmas antagónicos. Estudios Pedagógicos, vol. 39, núm. 1, pp. 377-391. http://dx.doi.org/10.4067/S071807052013000100022

Morales, M. y López, V. (2019). Políticas de convivencia escolar en América Latina: cuatro perspectivas de comprensión y acción. Archivos Analíticos de Políticas Educativas, vol. 2, núm. 5, pp.1-28. http://dx.doi.org/10.14507/epaa.27.3800 Parra, L. (2016). Transformación creativa de los conflictos. En V. Ocejo y A. P. Hernández (coords.). Derechos humanos y educación para la paz. México: Universidad Iberoamericana, AC.

Pranis, K., Stewart, B. \& Wedge, M. (2003). Peacemaking circles. From conflict to community. EUA: Living Justice Press.

PREAL (2003). Estrategias para la prevención de la violencia y promoción de una cultura de paz en las escuelas. Serie Prevención de la Violencia Escolar, vol. 1, núm. 1. Promoción de la Reforma Educativa en América Latina y el Caribe. 
Pulido, R., Martín-Seoane, G. y Lucas-Molina, B. (2013). Orígenes de los programas de mediación escolar: distintos enfoques que influyen en esta práctica restaurativa. Anales de Psicología, vol. 29, núm. 2, pp. 385-392. https://doi. org/10.6018/analesps.29.2.132601

SEP (2017). Programa Nacional de Convivencia Escolar. Protocolos para la detección, prevención y actuación en situaciones de abuso sexual infantil, acoso escolar y maltrato en las escuelas de educación inicial y básica para el estado de Guanajuato. México. http://www.seg.guanajuato.gob.mx/AConvivir/Paginas/dctos/Protocolo/Protocolo_Guanajuato.pdf

Villanueva-Badenes, L., Usó-Guiral, I. y Adrián-Serrano, J. E. (2013). Los programas de mediación entre iguales: una herramienta eficaz para la convivencia escolar. Apuntes de Psicología, vol. 31, pp. 165-171. http://www.apuntesdepsicologia.es/index.php/revista/article/view/319

Zurita-Rivera, U. (2013). Políticas, programas, legislación, proyectos y acciones gubernamentales y no gubernamentales. En A. Furlan y T. C. Spitzer Schwartz (coord. gral.). Convivencia, disciplina y violencia en las escuelas 2002 -2011 (pp. 457-520). México: COMIE/ANUIES. 